\title{
Concerning Two Series for the Gamma Function
}

\author{
By John W. Wrench, Jr.
}

1. Introduction. It does not seem to be widely recognized that the Stirling asymptotic series for $\Gamma(x)$ yields accurate values for small integer arguments. However, Salzer [1] has pointed out the effectiveness of this series in approximating $\Gamma(z)$ for large values of $|z|$, even when $R(z)$ is quite small. Although the Stirling series for $\ln \Gamma(z)$ contains only odd powers of $z^{-1}$, whereas the corresponding series for $\Gamma(z)$ contains all powers of $z^{-1}$, nevertheless the latter provides an effective computational tool for the direct evaluation of $\Gamma(z)$, especially by means of modern digital computers.

For that reason, the exact (rational) values of the first twenty coefficients of Stirling's asymptotic series for $\Gamma(z)$ have been calculated and are tabulated herein.

The second series here considered is the power series for the entire function $1 / \Gamma(z)$. The first extensive calculation of the coefficients of this series appears to have been performed by Bourguet [2]. His $16 \mathrm{D}$ approximations were subsequently recalculated and corrected by Isaacson and Salzer [3]. These emended values have been reproduced in Davis [4] and in the NBS Handbook [5]. In the course of checking [6] these corrected values the present author has now recalculated these coefficients anew and extended the approximations to 31D. These new data are also tabulated in this paper, and their application is illustrated through the evaluation of the main minimum of $\Gamma(x)$ to $31 \mathrm{D}$.

2. Stirling's Asymptotic Series for $\Gamma(z)$. The coefficients of the Stirling series for $\Gamma(z)$ can be derived as follows. Let

$$
\Gamma(z)=(2 \pi / z)^{1 / 2} z^{2} e^{-z} G(z) .
$$

Then logarithmic differentiation yields

$$
\Gamma^{\prime}(z) / \Gamma(z)=-1 /(2 z)+\ln z+G^{\prime}(z) / G(z) .
$$

Next, we apply logarithmic differentiation to the Stirling series for $\ln \Gamma(z)$; namely,

$$
\ln \Gamma(z) \sim\left(z-\frac{1}{2}\right) \ln z-z+\frac{1}{2} \ln (2 \pi)+\frac{B_{2}}{1 \cdot 2 z}+\frac{B_{4}}{3 \cdot 4 z^{3}}+\frac{B_{6}}{5 \cdot 6 z^{5}}+\cdots,
$$

where $B_{2}=1 / 6, B_{4}=-1 / 30, B_{6}=1 / 42, \cdots$ are the Bernoulli numbers. We thereby obtain the following well-known series for the psi function, which also can be obtained directly by means of Watson's lemma [7]:

$$
\psi(z)=\Gamma^{\prime}(z) / \Gamma(z) \sim-\frac{1}{2 z}+\ln z-\frac{B_{2}}{2 z^{2}}-\frac{B_{4}}{4 z^{4}}-\frac{B_{6}}{6 z^{6}}-\cdots .
$$

Comparing this expansion with that in (2), we infer that

Received December 20, 1967. 


$$
G^{\prime}(z) / G(z) \sim-\frac{B_{2}}{2 z^{2}}-\frac{B_{4}}{4 z^{4}}-\frac{B_{6}}{6 z^{6}}-\cdots .
$$

We next assume that

$$
G(z) \sim 1+\frac{c_{1}}{z}+\frac{c_{2}}{z^{2}}+\frac{c_{3}}{z^{3}}+\cdots
$$

and that $G^{\prime}(z)$ also possesses an asymptotic expansion; then

$$
G^{\prime}(z) \sim-\frac{c_{1}}{z^{2}}-\frac{2 c_{2}}{z^{3}}-\frac{3 c_{3}}{z^{4}}-\cdots .
$$

By Eq. (5) we can then write

$$
G^{\prime}(z) \sim\left(-\frac{B_{2}}{2 z^{2}}-\frac{B_{4}}{4 z^{4}}-\frac{B_{6}}{6 z^{6}}-\cdots\right)\left(1+\frac{c_{1}}{z}+\frac{c_{2}}{z^{2}}+\cdots\right) .
$$

Expanding the product and comparing coefficients of like powers of $z^{-1}$ in (7), we obtain the recurrence formulas

$$
\begin{aligned}
(2 k-1) c_{2 k-1} & =\frac{B_{2}}{2} c_{2 k-2}+\frac{B_{4}}{4} c_{2 k-4}+\cdots+\frac{B_{2 k}}{2 k}, \\
2 k c_{2 k} & =\frac{B_{2}}{2} c_{2 k-1}+\frac{B_{4}}{4} c_{2 k-3}+\cdots+\frac{B_{2 k}}{2 k} c_{1},
\end{aligned}
$$

where $k=1,2,3, \cdots$, and $c_{0}=1$.

Since

$$
B_{2 n}=(-1)^{n+1}(2 n) ! 2^{-2 n+1} \pi^{-2 n} \zeta(2 n),
$$

we infer that $\left|B_{2 n} / B_{2 n-2}\right| \sim n\left(n-\frac{1}{2}\right) / \pi^{2}$, and therefore

$$
\begin{aligned}
c_{2 k-1} & \sim B_{2 k} / 2 k(2 k-1), \\
c_{2 k} & \sim B_{2 k} c_{1} /(2 k)^{2}=B_{2 k} /\left(48 k^{2}\right) .
\end{aligned}
$$

Consequently, for large $k, c_{2 k} \approx c_{2 k-1} / 12$. Furthermore, we observe from (3) that $B_{2 k} / 2 k(2 k-1)$ is simply the coefficient of $z^{-2 k+1}$ in Stirling's series for $\ln \Gamma(z)$. It is interesting to note that the decimal values of $c_{2 k-1}$ agree to at least two or three significant figures with those of $B_{2 k} / 2 k(2 k-1)$ for $k=1(1) 15$. This comparison was facilitated by the extensive decimal table of the latter coefficients calculated by Uhler [8].

F. D. Murnaghan and this writer [9] have derived asymptotic series for the coefficients $c_{i}$, of which the leading terms are

$$
\begin{aligned}
& c_{2 k+1} \sim(-1)^{k}\left[1-\frac{3}{2(4 k-1)}+\cdots\right] \psi_{2 k+1}, \\
& c_{2 k+2} \sim(-1)^{k}\left[\frac{1}{3(4 k+1)}-\frac{5}{6(4 k+1)(4 k-1)}+\cdots\right] \pi \psi_{2 k+2},
\end{aligned}
$$

where

$$
\psi_{j}=\frac{(2 j-3) ! !(2 j+1) ! !}{2^{2 j} \pi^{j}(2 j) ! !}
$$




\section{TABLE 1}

The first ticenty coefficients in the Stirling asymptotic series for $\Gamma(z)$

$$
\begin{array}{ll}
c_{0} \quad 1 \\
c_{1} \quad \frac{1}{12} \\
c_{2} \quad \frac{1}{288} \\
c_{3}-\frac{139}{\overline{5} 1840} \\
c_{4}-\frac{571}{2488320} \\
c_{5} \quad \frac{163,879}{209,018,880} \\
c_{6} \quad \frac{5,246,819}{75,246,796,800} \\
c_{7}-\frac{534,703,531}{902,961,561,600} \\
c_{8}-\frac{4,483,131,259}{86,684,309,913,600}
\end{array}
$$

$c_{9} \quad 432,261,921,612,371$

$\overline{514,904,800,886,784,000}$

$c_{10} \quad 6,232,523,202,521,089$

$\overline{86,504,006,548,979,712,000}$

$c_{11}-25,834,629,665,134,204,969$

$13,494,625,021,640,835,072,000$

$c_{12}-1,579,029,138,854,919,086,429$

$9,716,130,015,581,401,251,840,000$

$c_{13} \quad 746,590,869,962,651,602,203,151$

$\overline{116,593,560,186,976,815,022,080,000}$

$c_{14} \quad 1,511,513,601,028,097,903,631,961$

$2,798,245,444,487,443,560,529,920,000$

$c_{15}-8,849,272,268,392,873,147,705,987,190,261$

$299,692,087,104,605,205,332,754,432,000,000$

$c_{16}-142,801,712,490,607,530,608,130,701,097,701$

$57,540,880,724,084,199,423,888,850,944,000,000$

$c_{17} \quad 2,355,444,393,109,967,510,921,431,436,000,087,153$

$13,119,320,805,091,197,468,646,658,015,232,000,000$

$c_{18} \quad 2,346,608,607,351,903,737,647,919,577,082,115,121,863$

$155,857,531,164,483,425,927,522,297,220,956,160,000,000$

$c_{19}-\frac{2,603,072,187,220,373,277,150,999,431,416,562,396,331,667}{1,870,290,373,973,801,111,130,267,566,651,473,920,000,000}$

$c_{20}-73,239,727,426,811,935,976,967,471,475,430,268,695,630,993$ $628,417,565,655,197,173,339,769,902,394,895,237,120,000,000$ 


\section{TABLE 2}

$50 \mathrm{D}$ values of the first twenty coefficients in the asymptotic series for $\Gamma(z)$

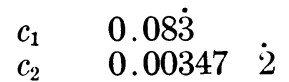

$c_{3}-0.00268 \quad 13 \dot{2} 71 \quad 60493 \dot{8}$

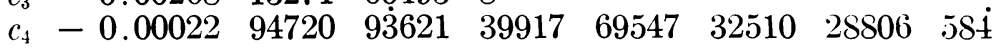

$\begin{array}{lllllllllll}c_{5} & 0.00078 & 40392 & 21720 & 06662 & 74740 & 34881 & 44228 & 88496 & 96257 & 10366\end{array}$

$\begin{array}{lllllllllll}c_{6} & 0.00006 & 97281 & 37583 & 65857 & 77429 & 39882 & 85757 & 83308 & 29359 & 63594\end{array}$

$\begin{array}{lllllllllll}c_{7} & -0.00059 & 21664 & 37353 & 69388 & 28648 & 36225 & 60440 & 11873 & 91585 & 19680\end{array}$

$\begin{array}{lllllllllll}c_{8} & -0.00005 & 17179 & 09082 & 60592 & 19337 & 05784 & 30020 & 58822 & 81785 & 34534\end{array}$

$\begin{array}{lllllllllll}c_{9} & 0.00083 & 94987 & 20672 & 08727 & 99933 & 57516 & 76498 & 34451 & 98182 & 11159\end{array}$

$\begin{array}{lllllllllll}c_{10} & 0.00007 & 20489 & 54160 & 20010 & 55908 & 57193 & 02250 & 15052 & 06345 & 17380\end{array}$

$\begin{array}{llllllllll}c_{11}-0.00191 & 44384 & 98565 & 47752 & 65008 & 98858 & 32852 & 25448 & 76893 & 57895\end{array}$

$\begin{array}{lllllllllll}c_{12}-0.00016 & 25162 & 62783 & 91581 & 68986 & 35123 & 98027 & 09981 & 05872 & 59193\end{array}$

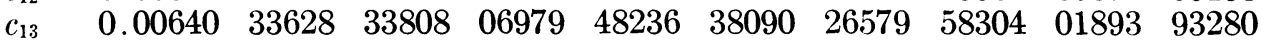

$\begin{array}{llllllllllll}c_{14} & 0.00054 & 01647 & 67892 & 60451 & 51804 & 67508 & 57024 & 17355 & 47254 & 41598\end{array}$

$\begin{array}{llllllllll}c_{15}-0.02952 & 78809 & 45699 & 12050 & 54406 & 51054 & 69382 & 44465 & 65482 & 82544\end{array}$

$\begin{array}{lllllllllll}c_{16}-0.00248 & 17436 & 00264 & 99773 & 09156 & 58368 & 74346 & 43239 & 75168 & 04723\end{array}$

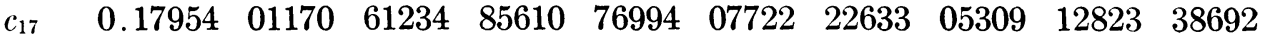

$\begin{array}{lllllllllll}c_{18} & 0.01505 & 61130 & 40026 & 42441 & 23842 & 21877 & 13112 & 72602 & 59815 & 45541\end{array}$

$\begin{array}{llllllllll}c_{19}-1.39180 & 10932 & 65337 & 48139 & 91477 & 63542 & 27314 & 93580 & 45617 & 72646\end{array}$

$\begin{array}{lllllllllll}c_{20}-0.11654 & 62765 & 99463 & 20085 & 07340 & 36907 & 14796 & 96789 & 37334 & 38371\end{array}$

Note. The first four entries are shown as repeating decimals, the repetends being indicated by superior dots.

We infer from these relations that

$$
c_{2 k} / c_{2 k-1} \sim \frac{1}{12}\left(1+\frac{1}{4 k+1}\right),
$$

which refines our previous estimate of the relative magnitudes of $c_{2 k}$ and $c_{2 k-1}$.

By means of Eqs. (9) and (10), the tabulated exact values of the coefficients $c_{i}$ were successively calculated for $i=1(1) 20$. The accuracy of the first nine coefficients as published by Davis [10] is confirmed.

For ease in application to specific calculations, a table of $50 \mathrm{D}$ equivalents of these exact coefficients is also included. (The first four entries are shown as repeating decimals in this range.) Several entries beyond the range of this table were evaluated [9] to 6 or 7S by the asymptotic series (14) and (15). For completeness, these supplementary values are reproduced here in Table 3.

\section{TABLE 3}

Supplementary values of the coefficients in the asymptotic series for $\Gamma(z)$

$\begin{array}{cc}j & c_{j} \\ 21 & 13.3980 \\ 22 & 1.12093 \\ 23 & -156.802 \\ 24 & -13.1088 \\ 25 & 2192.56 \\ 26 & 183.199 \\ 27 & -36101.1 \\ 28 & -3015.17 \\ 29 & 691346.4 \\ 30 & 57722.53\end{array}$


3. Power Series for $1 / \Gamma(z)$. We start with Legendre's series

$$
\ln \Gamma(1-z)=\gamma z+\sum_{k=2}^{\infty} S_{k} z^{k} / k, \quad|z|<1,
$$

where $\gamma$ is Euler's constant and $S_{k}=\zeta(k)=\sum_{k=1}^{\infty} n^{-k}$. This series in combination with the reflection formula

$$
\Gamma(z) \Gamma(1-z)=\pi / \sin \pi z, \quad 0<R(z)<1
$$

and the series

$$
\ln \left(\frac{\sin \pi z}{\pi z}\right)=-\sum_{k=1}^{\infty} S_{2 k} z^{2 k} / k
$$

yields the series

$$
\ln [z \Gamma(z)]=-\gamma z+\sum_{k=2}^{\infty}(-1)^{k} S_{k} z^{k} / k, \quad|z|<1,
$$

from which we infer

$$
\frac{1}{\Gamma(z)}=z \exp \left\{\gamma z-\sum_{k=2}^{\infty}(-1)^{k} S_{k} z^{k} / k\right\},
$$

whence we obtain

$$
\frac{1}{\Gamma(z)}=\sum_{k=1}^{\infty} a_{k} z^{k}
$$

where

$$
a_{1}=1, \quad a_{2}=\gamma
$$

and

$$
i a_{i}=\gamma a_{i-1}-S_{2} a_{i-2}+S_{3} a_{i-3}-\cdots+(-1)^{i+1} S_{i} \quad(i \geqq 2) .
$$

This recurrence formula was originally derived by Bourguet [2] in a different manner, starting with Euler's infinite product for $\Gamma(z)$.

Since

$$
\ln (1+z)=\sum_{k=1}^{\infty}(-1)^{k+1} z^{k} / k, \quad|z|<1,
$$

we infer that Eq. (20) is equivalent to

$$
\ln [z(1+z) \Gamma(z)]=(1-\gamma) z+\sum_{k=2}^{\infty}(-1)^{k} S_{k}{ }^{\prime} z^{k} / k,
$$

where $S_{k}{ }^{\prime}=\zeta(k)-1=\sum_{n=2}^{\infty} n^{-k}$.

Hence, if we write

$$
\frac{1}{\Gamma(z)}=z(1+z)\left[b_{0}+b_{1} z+b_{2} z^{2}+\cdots\right],
$$

we find 
(26)

$$
\begin{gathered}
b_{0}=1, \quad b_{1}=\gamma-1, \\
i b_{i}=(\gamma-1) b_{i-1}-S_{2}{ }^{\prime} b_{i-2}+S_{3}{ }^{\prime} b_{i-3}-\cdots+(-1)^{i+1} S_{i}{ }^{\prime} \quad(i \geqq 2) .
\end{gathered}
$$

Moreover, the coefficients in series (22) and (25) are connected by the relation

$$
a_{i}=b_{i-1}+b_{i-2}, \quad(i \geqq 2) .
$$

It was found more convenient to calculate the coefficients $b_{i}$ in succession from Eq. (26) and then to deduce the corresponding values of $a_{i}$ from Eq. (27).

Several omnibus checks have been applied to the tabulated values $a_{i}{ }^{*}, b_{i}{ }^{*}$ of the coefficients $a_{i}$ and $b_{i}$. These results are

$$
\begin{gathered}
\sum_{i=1}^{41} a_{i}{ }^{*}=1+4 \cdot 10^{-31}, \\
\sum_{i=0}^{39} b_{i}{ }^{*}=\frac{1}{2}+2 \cdot 10^{-31}, \\
\sum_{i=1}^{34} a_{i}{ }^{*} / 2^{i}=\pi^{-1 / 2}+2 \cdot 10^{-32} .
\end{gathered}
$$

A further partial check on the accuracy of these approximations to $a_{i}$ was made possible through the kindness of Yudell L. Luke, who sent the author an unpublished table of these coefficients calculated to about $28 \mathrm{D}$ at Midwest $\mathrm{Re}-$ search Institute by Rosemary Moran under his direction. Agreement of these results with those of the author to at least $27 \mathrm{D}$ was noted.

\begin{tabular}{crrrrrrrr}
\multicolumn{7}{c}{ TABLE 4} \\
$i$ & \multicolumn{7}{c}{ Coefficients $b_{i}$ to 31D } \\
1 & -0.42278 & 43350 & 98467 & 13939 & 34879 & 09917 & 6 \\
2 & -0.23309 & 37364 & 21786 & 74168 & 35316 & 05227 & 8 \\
3 & 0.19109 & 11013 & 87691 & 50615 & 45276 & 70352 & 4 \\
4 & -.02455 & 24900 & 05400 & 01665 & 28268 & 75250 & 3 \\
5 & -.01764 & 52445 & 50144 & 32009 & 53814 & 26038 & 9 \\
6 & .0802 & 32730 & 22267 & 34653 & 32665 & 04366 & 6 \\
7 & -.080 & 43297 & 75604 & 24699 & 08714 & 94026 & 1 \\
8 & -.036 & 08378 & 16254 & 81812 & 12424 & 77057 & 9 \\
9 & 3 & .014 & 55961 & 42139 & 86714 & 84267 & 47094 & 8 \\
10 & -.01 & 75458 & 59751 & 75096 & 22735 & 48468 & 5 \\
11 & -.0 & 25889 & 95029 & 03727 & 63821 & 40922 & 9
\end{tabular}


12

13

14

15

16

17

18

19

20

21

22

23

24

25

26

27

28

29

30

31

32

33

34

35

36

37

38

39 $\begin{array}{lllllll}.0 & 13385 & 01546 & 89460 & 57247 & 95563 & 4\end{array}$

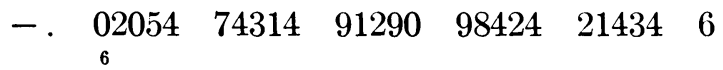

$\begin{array}{cccccc}-.01 & 59526 & 78485 & 08679 & 23581 & 3\end{array}$

$\begin{array}{llllll}.062 & 75621 & 88933 & 22837 & 41444 & 7\end{array}$

$\begin{array}{cccccc}-.012 & 73614 & 24486 & 30608 & 11388 & 5\end{array}$

$\begin{array}{llllll}.0 & 92339 & 67437 & 60406 & 66800 & 2\end{array}$

$\begin{array}{llllll}.0 & 12002 & 99679 & 30693 & 84248 & 6\end{array}$

$\begin{array}{rrrrrr}10 & & & & & \\ \text {-. } & 04220 & 73335 & 31643 & 12994 & 9\end{array}$

$\begin{array}{llllll}11 & & & & & \\ 0 & 0523 & 92773 & 45221 & 07286 & 7\end{array}$

$\begin{array}{lllll}.0523 & 92773 & 45221 & 07286 & 7\end{array}$

$\begin{array}{ccccc}-.013 & 89070 & 57766 & 59688 & 8\end{array}$

$\begin{array}{ccccc}-.06 & 69255 & 47590 & 05379 & 1\end{array}$

$\begin{array}{lllll}.01 & 34443 & 22195 & 82361 & 1\end{array}$

$\begin{array}{ccccc}-.0 & 11765 & 35913 & 44100 & 2\end{array}$

$\begin{array}{cccc}-.047 & 23388 & 25645 & 4\end{array}$

$\begin{array}{llll}.0165 & 90310 & 80397 & 1\end{array}$

$\begin{array}{llll}17 & & & \\ -.024 & 66504 & 25079 & 1\end{array}$

$\begin{array}{llll}18 & 01 & 67758 & 5663 \% \quad \text { ј }\end{array}$

$19 \quad 03682 \quad 06583 \quad 8$

$\begin{array}{lll}03682 & 06583 & \mathrm{~S}\end{array}$

-. $\begin{array}{lll}02344 & 71410 & 7\end{array}$

$.0290 \quad 48055 \quad 6$

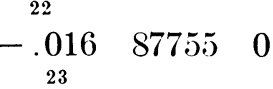

$\begin{array}{rrr}-.0 & 44601 & 4 \\ 25 & & \end{array}$

$\begin{array}{lll}.0 & 20995 & 4\end{array}$

-. $\begin{array}{lll}02345 & 4 \\ 26 & \end{array}$

$\begin{array}{ll}.0127 & 4 \\ 27 & \end{array}$

.025

$-.01 \quad 3$

$\begin{array}{rr}.0 & 1 \\ 30 & \end{array}$ 
4. The Main Minimum of $\Gamma(x)$. As an example of a nontrivial application of Table 5, the main minimum of the factorial function $x$ !, or $\Gamma(1+x)$, has been evaluated thereby to $31 \mathrm{D}$.

The abscissa of this minimum was calculated from the equation

$$
\begin{aligned}
\psi(x) & =\frac{\Gamma^{\prime}(1+x)}{\Gamma(1+x)} \\
& =\frac{1}{2 x}-\frac{1}{1-x^{2}}-\frac{\pi}{2} \cot \pi x+(1-\gamma)-S_{3} x^{2}-S_{5}^{\prime} x^{4}-\cdots=0,
\end{aligned}
$$

starting with a $15 \mathrm{D}$ approximation due to J. C. P. Miller [11] and applying NewtonRaphson iteration. The required abscissa to $33 \mathrm{D}$ was thus found to be

$$
x_{0}=0.46163 \quad 21449 \quad 68362 \quad 34126 \quad 26595 \quad 42325 \quad 721 \quad \cdots \text {. }
$$

Then $1 / \Gamma\left(1+x_{0}\right),=1 / x_{0} \Gamma\left(x_{0}\right)$, was evaluated from series (22), using synthetic division and the coefficients in Table 5. This calculation yielded the approximation

$$
1 / \Gamma\left(1+x_{0}\right)=1.12917 \quad 38854 \quad 50141 \quad 23991 \quad 36073 \quad 09471 \quad 1 \cdots,
$$

whose reciprocal is

$$
\Gamma\left(1+x_{0}\right)=0.88560 \quad 31944 \quad 10888 \quad 70027 \quad 88159 \quad 00582 \quad 6 \cdots .
$$

This confirms the accuracy of the $15 \mathrm{D}$ approximation given by Miller.

TABLE 5

Coefficients $a_{i}$ to $31 \mathrm{D}$

$a_{i}$

$\begin{array}{llllllll}2 & 0.57721 & 56649 & 01532 & 86060 & 65120 & 90082 & 4\end{array}$

$\begin{array}{llllllll}3 & -0.65587 & 80715 & 20253 & 88107 & 70195 & 15145 & 4\end{array}$

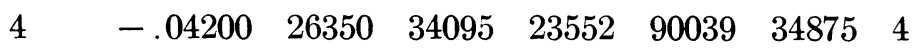

$\begin{array}{lllllllll}5 & .16653 & 86113 & 82291 & 48950 & 17007 & 95102 & 1\end{array}$

$\begin{array}{lllllllll}6 & -.04219 & 77345 & 55544 & 33674 & 82083 & 01289 & 2\end{array}$

$7 \quad-.0962 \quad 19715 \quad 27876 \quad 97356 \quad 21149 \quad 21672 \quad 3$

$\begin{array}{llllllll}8 & .0721 & 89432 & 46663 & 09954 & 23950 & 10340 & 5\end{array}$

$9 \quad-\quad \begin{array}{lllllllll}2 & 0116 & 51675 & 91859 & 06511 & 21139 & 71084 & 0\end{array}$

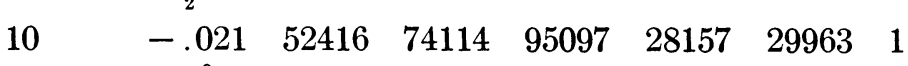

$\begin{array}{lllllllll}11 & .012 & 80502 & 82388 & 11618 & 61531 & 98626 & 3\end{array}$

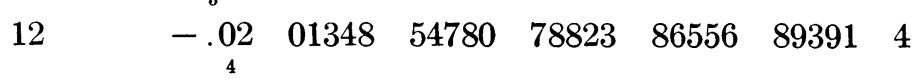

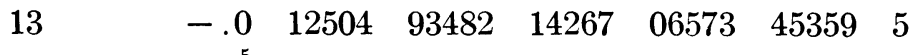


$\begin{array}{lllllll}.0 & 11330 & 27231 & 98169 & 58823 & 74128 & 9 \\ 5 & & & & & \end{array}$

18

-. $\begin{array}{cccccc}02056 & 33841 & 69776 & 07103 & 45015 & 9\end{array}$ $\begin{array}{llllll}.061 & 16095 & 10448 & 14158 & 17863 & 4\end{array}$ $\begin{array}{llllll}8 & 050 & 02007 & 64446 & 92229 & 30056\end{array}$ $\begin{array}{llllll}.050 & 02007 & 64446 & 92229 & 30056 & 2\end{array}$ $\begin{array}{cccccc}-.011 & 81274 & 57048 & 70201 & 44588 & 3\end{array}$

$\begin{array}{cccccc}.01 & 04342 & 67116 & 91100 & 51048 & 8\end{array}$

22

23

24

25

26

27

28

29

30

31

32

$\begin{array}{llllll}\text {. } & 07782 & 26343 & 99050 & 71253 & 7\end{array}$ $\begin{array}{lllll}11 & & & & \\ 03696 & 80561 \quad 86422 \quad 05708 \quad 2\end{array}$

-. $03696 \quad 80561 \quad 86422 \quad 05708 \quad 2$ $\begin{array}{llllll}.0510 & 03702 & 87454 & 47597 & 9\end{array}$ 12 $\begin{array}{ccccc}-.020 & 58326 & 05356 & 65067 & 9\end{array}$

$\begin{array}{ccccc}-.05 & 34812 & 25394 & 23018 & 0\end{array}$ $\begin{array}{lllll}.01 & 22677 & 86282 & 38260 & 9\end{array}$ $\begin{array}{ccccc}-.0 & 11812 & 59301 & 69745 & 6\end{array}$ $\begin{array}{llll}.0118 & 66922 & 54751 & 7\end{array}$

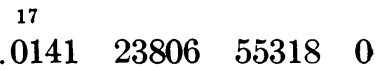
$\begin{array}{llll}.0141 & 23806 & 55318 & 0\end{array}$ $\begin{array}{cccc}-.022 & 98745 & 68443 & 6\end{array}$ $\begin{array}{llll}.01 & 71440 & 63219 & 3\end{array}$ $\begin{array}{llll}\text {. } & 01337 & 35173 & 1\end{array}$ - 21337351731

33

-. 02054233551 21

$.027360300 \quad 6$ 22

34

$\begin{array}{ccc}-.017 & 32356 & 4\end{array}$

35

$\begin{array}{ccc}-.0 & 23606 & 0\end{array}$

36

$\begin{array}{lll}.0 & 18650 & 0\end{array}$

37

- 022180

38

.01299

39

.012

40

$\begin{array}{rr}-.01 & 1 \\ 29 & \end{array}$ 
Applied Mathematics Laboratory

Naval Ship Research and Development Center

Washington, D. C. 20007

1. Table of the Gamma Function for Complex Arguments, Nat. Bur. Standards Appl. Math. Series, 34, U. S. Government Printing Office, Washington, D. C., 1954, p. viii. MR 16, 403.

2. L. Bourguet, "Sur les intégrales Euleriennes et quelques autres fonctions uniformes," Acta Math., v. 2, 1883, pp. 261-295.

3. $M T A C$, v. 1,1943 . p. 124, MTE 19.

4. H. T. Davis, Tables of the Mathematical Functions, Vol. I, Trinity Univ. Press, Texas, 1963, p. 185.

5. Milton Abramowitz \& I. A. Stegun, (Editors), Handbook of Mathemalical Functions with Formulas, Graphs, and Mathematical Tables, Nat. Bur. Standards Appl. Math. Series, 55, Superintendent of Documents, U. S. Government Printing Office, Washington, D. C., 1964, p. 256; 3rd printing, with corrections, 1965. MR 29 \#4914; MR 31 \#1400.

6. Math. Comp., v. 20, 1966, p. 641, MTE 399. (The final three digits in the 28D value of $c_{23}$ given in the editorial footnote should read 650 instead of 479.)

7. E. T. Copson, Asymptotic Expansions, Cambridge Tracts in Mathematics and Mathematical Physics, No. 55, Cambridge Univ. Press, New York, 1964, pp. 51-52. MR 29 \#6234.

8. H. S. Uhler, "The coefficients of Stirling's series for $\log \Gamma(x)$," Proc. Nat. Acad. Sci. U.S.A., v. 28, 1942, pp. 59-62. MR 3, 275.

9. F. D. Murnaghan \& J. W. Wrench, JR., The Converging Factor for the Exponential Integral, David Taylor Model Basin Report 1535, 1963.

10. H. T. Davis, loc. cit., pp. 180-181.

11. A. Fletcher, J. C. P. Miller, L. Rosenhead \& L. J. Comrie, An Index of Mathematical T'ables, Vol. I, 2nd ed., Addison-Wesley, Reading, Mass., 1962, p. 295. MR 26 \#365a. 